\title{
The Research on Chinese Idioms Educational Games in TCFL Based on Virtual Reality
}

\author{
Xiao-Qiang HU, Rui SUa, and Ling HE \\ Jiangxi Science \& Technology Normal University, 330038 Nanchang, China \\ a Corresponding author: suruigx1990@163.com
}

\begin{abstract}
In recent years, overseas set off a craze for learning Chinese. Chinese Idioms teaching is important but difficult for foreigners in Teaching Chinese as a Foreign Language (TCFL). The VR educational game should be a good choice to solve the problem with education, entertainment, and high immerse. In this study, 'Yu Gong moves the mountain' is designed as an example. Unity 3D is the main development tool, Leap Motion is interactive way, and also doing an interview with some findings and conclusions. Comparison of textbooks or teachers explaining, VR Chinese Idioms educational game can enhance most of the learners understanding with intense interest. However, the educational game should be integrated into more entertainment and it costs at least 10 minutes or more to adapt the operation of Leap Motion. In the further research, the VR Chinese Idioms educational games for the TCFL can be better gradually based on this research.
\end{abstract}

\section{Introduction}

In recent years, overseas set off a craze for learning Chinese. Development of TCFL is meaningful for promoting Chinese, spreading the excellent culture of the China and enhancing mutual understanding between the Chinese and abroad. What's more, it is significance to enhance China's influence in the world by TCFL. Chinese Idioms are formed in the long-term practice of Chinese people's life. They are usually made of fixed phrase and fixed structure with special cultural connotations. So that, foreign students whose mother language isn't Chinese often think that the vocabulary and emphases in Chinese idioms are real difficulties to get. In fact, most Chinese idioms are generated from the historical story. If new media and teaching theory could be used in Chinese Idioms teaching of TCFL, learners will understand the meaning of Chinese Idioms directly with more fun.

In 2015, VR is developing rapidly, both hardware and software. With rapid popularization of VR, it brings unprecedented convenience and entertainment to human's daily life. The applications of VR have been utilized into many research fields, such as medicine, entertainment, military, electronic commerce and so on. In recent years, with combination of education, VR becomes a widespread focus. The educational games based on VR technology are more entertainment and immersed than normal ones, especially natural human-computer interaction. That's the reason why the educational games are an effective to combine VR and education.

An educational game for Chinese Idioms in TCFL based on VR Technology is designed. After that, some foreign learners are invited to test it. The conclusion of the is shown at the end of this paper.

\section{Related Work}

\subsection{The Related Work of Chinese Idioms Teaching in TCFL}

Chinese Idioms teaching plays an important role in TCFL to foreign learners. No only expanding their vocabulary, but also improving their ability to express and reading. And it helps them understand Chinese traditional culture. Because of the bias form, semantics, grammar, and pragmatics, foreign learners usually think Chinese Idioms learning is a difficulty. In traditional TCFL, teaching structure method, correction method and translation method are often adopted. In those methods, students can't teach in accordance with their aptitude with lower efficiency and effect.

Bo HONG (2003) points out that foreign students who are learning Chinese Idioms can't accurately understand the Chinese Idioms meaning. It's not clear idiomatic collocation, is also not clear about the usefulness of Chinese Idioms. He also analyzes bias in TCFL and thinks that that the Chinese idioms teaching should be focused on three points: the meaning of the Chinese Idioms teaching; Chinese Idioms pragmatic and collocation of idiom teaching.

Lina SHEN (2007) affirms teaching Chinese to foreign research results of TCFL in the ten years and predicts that idiom teaching research in TCFL tends to be diversified.

According to the source, Juan FENG (2011) analyses the characteristics of Chinese Idioms and the necessity for learning. And he comes out eight teaching methods in TCFL: function method, contrastive method between 
Chinese and English, animated graphics, performance realization method and so on.

Zhen XU (2013) proceed from the cultural introduction, put forward the necessity of reforming in Chinese idioms teaching method, improving its quality, and enhancing in TCFL.

\subsection{The Related Work of VR Educational Games}

In the game-based learning, learners have to learn by doing. Compared to traditional teaching, game-based learning is better with higher memory retention rate by $5 \%$. After experiencing the retention rate can be increased to $75 \%$. Watching, listening and doing at the same time, the retention rate of learners increases to $90 \%$ (Magennis \& Farrell, 2005). Junjie SHANG (2015), school of education, Peking University, believes that the real society is constantly merging with the virtual society, and the method of the virtual system is the solution to the real problem in the future. Game-based learning can create the immersive situation so that learners are more interested in learning and have the deep learning and get a better effect.

At present, the researchers have made some achievements of educational games in the TCFL. Ni ZHANG, Zhang YI, Kuiyuan ZHANG (2008) design the game platform, content, scene and plot contents, standing on the basis of empirical research, combining with the characteristics of learners for TCFL.

Based on the effective learning environment and the theory of multiple intelligences, Li CAI and Fangyu LIU (2011) proposes TCFL education game design model, according to game-based learning environment, characteristic and detailed design steps. They think about how to combine education and game, by designing characteristics, model designing, content and curriculum and making the example of DONGYOUJI.

After analyzing characteristics of the network game, Yang YI (2012) does some researches about network educational game for TCFL. Combined with the specific situation of the game, the possibility and advantage of network games in a foreign language are analyzed. And he tries to design the scripting tasks of network educational game for TCFL.

About VR educational game, Jianrong MA and Jingsu ZHANG (2012) design and development a parenting education game based on the Kinect. Jiali LIU has used the Unity3D game engine to achieve a child safety education. VR educational games are more applied in the field of early childhood education. There is any example in TCFL.

In this study, according to a combination of education and entertainment, VR Chinese Idioms educational games are design and tested. It could be a new form of edutainment in TCFL and also as an auxiliary means of TCFL. Through the Internet and other media, it can be extended to Chinese education for overseas students and Confucius Institute overseas.

\subsection{Main Process}

Methods in this study include the literature data method, interview, and observation method. The purpose is to designing and implication of VR Chinese Idioms educational games in TCFL. After designing the case, some learners are invited to test the VR Chinese Idioms educational game, interview contents they given will be analyzed, then coming to some conclusion. 20 learners who are non-Chinese speakers are selected to participate in the experiment. The flow chart of this research is as shown in Figure 1.

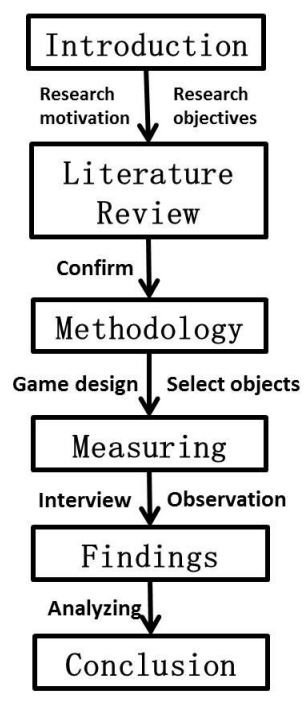

Figure 1. The flow chart in this research.

\subsection{Objects Selection}

There is an ancient Chinese fable 'The Foolish Old Man Who Removed the Mountains'. And it becomes an idiom that is named 'Yu Gong moves the mountain' reminds us that a person can never know what's possible unless he tries to make it happen. 'Yu Gong moves the mountains' is the case of the educational game based on VR for TCFL in this study.

Once upon a time, there is a very old man called $\mathrm{Yu}$ Gong. There are two mountains near his house. They are so high and big that it took a long time to walk to the other side. Yu Gong tells his family that they should all help him to move the mountains. And he said they could put the earth and stone into the sea because it's big enough to hold everything. So they start digging the next day. Yu Gong and his family began to move some of the earth and stone to the sea. One day, Zhi Sou see Yu Gong and his children when they are working on moving the mountains. He tells $\mathrm{Yu}$ Gong that he could never do it because he is old and weak. As soon as the Zhi Sou finished talking, $\mathrm{Yu}$ Gong said that his family could continue to move the mountains after his died. His family would live and grow, but the mountains could not get larger. So Yu Gong and his family keep on digging day after day and year after year. Finally, the god is so moved that he calls for two gods to move the mountains away.

\section{Methodology}




\subsection{Game Design}

Educational games design is not only to consider the education but also to consider the entertainment. Educational game designers have the plan to allow learners to complete some goals, and learners should achieve educational goals. The task design of educational games is shown in the following three tables.

Table 1. Previous tasks in educational game design.

\begin{tabular}{|c|c|c|c|}
\hline \multirow[b]{2}{*}{ Task 1} & Content & Level & Time \\
\hline & $\begin{array}{c}\text { Have } \\
\text { conversation } \\
\text { with Yu Gong }\end{array}$ & Simple & 2 mins \\
\hline \multicolumn{4}{|c|}{$\begin{array}{l}\text { Learning objectives: Understanding the reason for moving } \\
\text { mountain. }\end{array}$} \\
\hline \multicolumn{4}{|c|}{$\begin{array}{c}\text { Task describe: In a trip, you find that an old man is digging } \\
\text { a mountain with tools, feeling very strange, and then stepped } \\
\text { forward to ask the reason. }\end{array}$} \\
\hline \multirow[b]{2}{*}{ Task 2} & Content & Level & Time \\
\hline & $\begin{array}{c}\text { Have a look at } \\
\text { surroundings }\end{array}$ & Simple & 2 mins \\
\hline \multicolumn{4}{|c|}{$\begin{array}{l}\text { Learning objectives: Analysing feasibility of 'Yu Gong } \\
\text { moves the mountains'. }\end{array}$} \\
\hline \multicolumn{4}{|c|}{$\begin{array}{l}\text { Task describe: There are two mountains near his house. } \\
\text { They are so high and big that it took a long time to walk to } \\
\text { the other side. Yu Gong tells his family that they should all } \\
\text { help him to move the mountain. }\end{array}$} \\
\hline
\end{tabular}

Table 2. Middle tasks in educational game design.

\begin{tabular}{|c|c|c|c|}
\hline \multirow[b]{2}{*}{ Task 3} & Content & Level & Time \\
\hline & $\begin{array}{l}\text { Cheer Yu } \\
\text { Gong up }\end{array}$ & Medium & 3 mins \\
\hline \multicolumn{4}{|c|}{$\begin{array}{l}\text { Learning objectives: Understanding the spirit of 'Yu Gong } \\
\text { moves the mountains'. }\end{array}$} \\
\hline \multicolumn{4}{|c|}{$\begin{array}{l}\text { Task describe: One day, Zhi Sou sees Yu Gong and his } \\
\text { children when they are working on moving the mountains. } \\
\text { He tells Yu Gong that he could never do it because he is old } \\
\text { and weak. You should cheer Yu Gong up. }\end{array}$} \\
\hline \multirow[b]{2}{*}{ Task 4} & Content & Level & Time \\
\hline & $\begin{array}{l}\text { Help Yu Gong } \\
\text { move mountain }\end{array}$ & Difficulty & 5 mins \\
\hline \multicolumn{4}{|c|}{$\begin{array}{l}\text { Learning objectives: Analysis feasibility of moving the } \\
\text { mountain. }\end{array}$} \\
\hline \multicolumn{4}{|c|}{$\begin{array}{l}\text { Task describe: As soon as the Zhi Sou finished talking, Yu } \\
\text { Gong said that his family could continue to move the } \\
\text { mountains after he died. His family would live and grow, but } \\
\text { the mountains could not get bigger. }\end{array}$} \\
\hline
\end{tabular}

Table 3. Final tasks in educational game design.

\begin{tabular}{|c|c|c|c|}
\hline \multirow{2}{*}{ Task 5 } & Content & Level & Time \\
\cline { 2 - 4 } & $\begin{array}{c}\text { To have } \\
\text { Conversation } \\
\text { with Yu Gong }\end{array}$ & Difficulty & 5 mins \\
\hline Learning objectives: Summarize Yu Gong 's spirit and learn \\
how to tells others. \\
\hline \multicolumn{3}{|c|}{ Task describe: To explain the great determination and } \\
courage of Yu Gong to god and ask for the help. \\
\hline
\end{tabular}

\subsection{Scene creating}

The high immersive virtual learning environment can stimulate learners' interest, arise the enthusiasm of learners so that learners participate and immerse them actively. The modeling tool is Using $3 \mathrm{D}$, a Chinese traditional scene for the educational game is created, as shown in Figure 2.

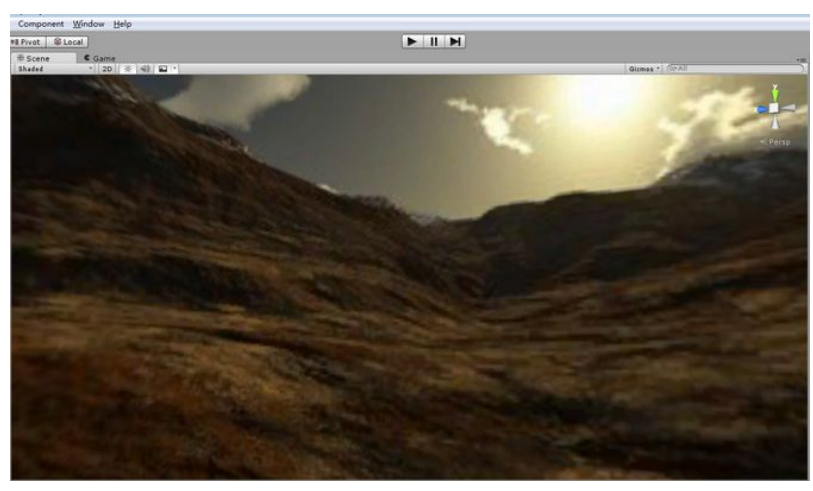

Figure 2. Scene creating of 'Yu Gong moves the mountain'.

Learners in the virtual environment when roaming through the rotation of the head of the action, to trigger the VR head wearing a display device in the mobile terminal of the gyroscope positioning function, learners will be able to observe the living environment of the ancients from 360 degrees different perspective.

\subsection{The interactive way}

The interactive way is a key part pf educational game design, including interface, NPC, controller and so on. Because VR belongs to the 3D media, it is not accurately by $2 \mathrm{D}$. In the traditional desktop virtual reality system, users can only rely on the mouse to move and other ways to change the viewing angle.In this study, Motion Leap is the input of the educational game learner, as shown in Fig.3.

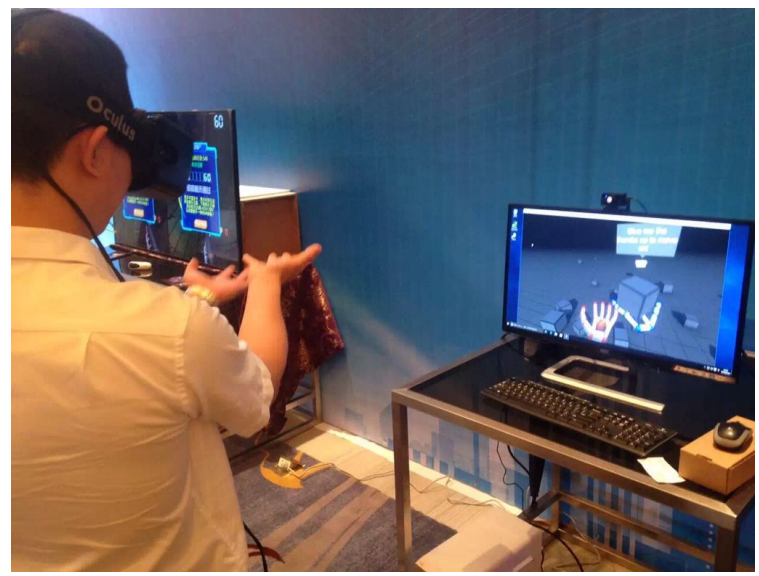

Figure 3. The interactive way of 'Yu Gong moves the mountains'.

The key part of code that makes combination with Unity 3D and Leap Motion is given: public class FingerMatch 
\{

static readonly float FingerBendState_Radian $=$

Mathf.PI*4f / 18 ;

static readonly float FingerStrightState_Radian $=$

Mathf.PI/12;

public static bool StrightState(FingerData fingerData,

float adjustBorder $=0 \mathrm{f}$ )

$\{$ bool isStright $=$ false;

Vector disalDir $=$ fingerData.m_Point.m_Direction;

if (!disalDir.Equals(Vector.Zero))

$\{$ Vector fingerDir $=$ fingerData.m_Point.m_Position -

fingerData.m_Position;

float radian = fingerDir.AngleTo(disalDir);

if (radian $<$ FingerStrightState_Radian + adjustBorder)

$\{$ isStright $=$ true; $\}\}$

return isStright;

public static bool BendState(FingerData fingerData, float

adjustBorder $=0 \mathrm{f} / /$, out float eulerAugle)

$\{$ bool isBend $=$ false;

Vector disalDir $=$ fingerData.m_Point.m_Direction;

if( !disalDir.Equals(Vector.Zero) )

$\{$ Vector fingerDir $=$ fingerData.m_Point.m_Position -

fingerData.m_Position;

float radian = fingerDir.AngleTo(disalDir);

if (radian $>$ FingerBendState_Radian + adjustBorder)

$\{$ isBend $=$ true; $\}$ \}

return isBend; $\}$

\}

Motion Leap can recognize the meaning of the user's gestures, and learners control the educational game as a natural human-computer interaction. In the case of the "Yu Gong moves the mountains", "Hands parallel expansion" on the leap Motion refers to moving in the educational game. If learners want to talk with the NPC, they should make a fist on the Leap Motion around the NPC. Scratching gesture stands for that mountain is bored. Users are helping Yu Gong to move the mountain.

\section{Conclusion}

After the development of Chinese Idioms Educational Games in TCFL, there are some findings. Comparison of textbooks or teachers explaining, VR Chinese Idioms educational game can enhance most of the learners understanding with intense interest. However, some feedbacks are negative. The educational game should be integrated into more entertainment. It costs at least 10 minutes or more to adapt the operation of Leap Motion.

According to current researches (Chinese Idioms teaching in TCFL and educational games), an idiom educational game for the TCFL based on VR is feasibility planning. In this study, 'Yu Gong moves the mountain' as a case is design and tested. After the interview, it should be improved, such as education and entertainment of the educational game are needed to be balanced. In the further research, the VR Chinese Idioms educational games for the TCFL can be better gradually. It will be an effective solution for promoting development in the TCFL.

\section{References}

1. Bo HONG, "Study on Chinese Idioms teaching in teaching Chinese as a second language," SUN YATSEN UNIVERSITY FORUM. Guangzhou. China, vol.23, pp. 297-300, February 2003.

2. Lina SHEN, "Summary of Chinese Idioms teaching in teaching Chinese as a second language in the past ten years," Journal of Language and Literature Studies,. Nanjing. China, vol 7, pp. 159-160, February 2007.

3. Juan FENG, The Discussion On Meaning And Teaching Method In Teaching Chinese As A Foreign Language.Wuhan,China: Central China normal university,2011.

4. Zhen XU, A Study on the Culture Lead-in in TCFL Chinese Idiom Teaching.Hangzhou,China: Zhejiang university,2013.

5. Junjie SHANG, "What is the most important value of virtual reality?"China Information Technology Education. Beijing. China, vol.10, pp.,October 2015.

6. Jianrong Ma,Sujing Zhang, "Parent-child interactive game design and implementation of technologybased somatosensory"China Electronic Education. Beijing. China, vol.09, pp.85-88,September 2015.

7. Xiaoqiang $\mathrm{HU}$, The Foundation and Application of Virtual Reality Technology. Beijing: BEIJING UNIVERSITY OF POSTS AND TELECOMMUCATIONS PRESS,2009. 